\section{AB1151 POLYMYALGIA RHEUMATICA. NEW THERAPEUTIC STRATEGY BASED ON LOW DOSE OF METROTEXATE PLUS LOCAL INFILTRATION WITH CORTICOSTEROIDS}

M. Retuerto Guerrero, E. Rodriguez, S. Melchor, N. Costas, J.L. Pablos, P. Fernández Dapica. Rheumatology, Hospital 12 de Octubre, Madrid, Spain

Background: The polymyalgia rheumatica is a rheumatic inflammatory disease more frequent in the eldest population The classic therapy is based on medium doses of corticosteroids followed by a maintenance phase of low doses, generally lasting form 1-2 years. Recurrences frequently require an escalation of dose, thus lengthening the treatment time, and that entails important comorbidity. Methotrexate (MTX) has been tested in 3 randomised clinical trials, showing in two of them the efficacy as a steroid sparing agent, but it has never been tested as monotherapy.

Objectives: To analyse the results of an alternative therapy in order to avoid the oral corticotherapy through the use of low dose of MTX and joint infiltration with a total duration of 24 months.

Methods: A prospective observational study in which patients that had been diagnosed with 2012 EULAR/ACR criteria were evaluated in outpatient medical consultations of Rheumatology at "Doce de Octubre" Hospital between 2015-2017, with the restriction of not having received previous steroid treatment. Right after diagnosis, the treatment with MTX $5-7.5 \mathrm{mg} / \mathrm{w}$ plus the infiltration of triamcinolone acetonide in both shoulders begins, being repeated if necessary after 15 or 30 days, or in case of subsequent relapse.

Results: 26 patients were included, with an average follow-up 19 \pm 5 months. ${ }^{12-24}$ The age average at diagnosis was $74 \pm 7$ years, ${ }^{55-83}$ being $56 \%$ women. $73 \%$ had symmetrical hand arthritis and $27 \%$ structural pathology of the rotator cuff. $96 \%$ had moderate-severe pain (VAS) in shoulders, $73 \%$ in hips and $54 \%$ in hands. These percentages after a month of treatment were $15 \%, 11.5 \%$ and $7.7 \%$. From the onset of symptoms until the start of treatment $104 \pm 54$ days passed, ${ }^{30-180}$ applying an initial dose of $5 \mathrm{mg}$ of MTX in half of cases and $7.5 \mathrm{mg}$ in the other half. There are no significant differences between precocity of the treatment or initial dose regarding a faster remission. The average of infiltrations in the shoulder per year is $2 \pm 1.4(1-4)$. $25 \%$ of patients showed reactivation with good response to the MTX dose increase [maximum dose $8 \pm 1.7(5-12.5)$ ] plus/or joint infiltration. The average time until the revision (subjective clinical evaluation, HAQ and APR) was $2.7 \pm 1$ months, ${ }^{1-6}$ being significantly higher $(p<0.05)$ in patients with peripheral arthritis $3.3 \pm 1.9$ vs $1.3 \pm 0.7$. The change of $\mathrm{HAQ}, \mathrm{CRP}$ and other variables are indicated in the table.

The treatment was stopped in $12 \%$ because of adverse effects (digestive intolerance, alopecia and respiratory infection in a patient with COPD). A patient showed mild plaquetopenia in wich not necessary to suspend the treatment with MTX. 8 patients were monitored during $\geq 2$ years, in $87 \%$ of them MTX has been removed with an average follow-up after the suspension of $3 \pm 1.7$ months ${ }^{1-6}$ with no need to restart it in any case.

Abstract AB1151 - Table 1

\begin{tabular}{lccccc}
\hline & Start & 1 Month & 3 Months & 6 Months & 12 Months \\
\hline Morning & $69 \%$ & $38 \%$ & $4 \%$ & $4 \%$ & $4 \%$ \\
stiffness $>1$ hour & & & & & \\
HAQ & 2 & 0.750 & 0.300 & 0.500 & 0.125 \\
ESR & 43,2 & 20 & 20 & 15 & 13 \\
CRP mg/dl $(0-0,5)$ & 4,1 & 0,81 & 0,44 & 0,48 & 0,47 \\
Peripheral arthritis & $77 \%$ & $23 \%$ & $7 \%$ & $11 \%$ & $7 \%$ \\
Shoulder limitation & $100 \%$ & $69 \%$ & $26 \%$ & $23 \%$ & $20 \%$ \\
\hline
\end{tabular}

Conclusions: The use of low dose of MTX and local joint infiltration with corticosteroids (initial and on demand) is an efficient therapeutic strategy with a low complication rate in PMR. All these results must be confirmed with controlled studies and a longer period of follow-up after the suspension of treatment.

Disclosure of Interest: None declared

DOI: 10.1136/annrheumdis-2018-eular.7172

\section{AB1152 MUSCULOSKELETAL MANIFESTATION OF DIABETES MELLITUS IS HIGHLY PREVALENT AND IS ASSOCIATED WITH POOR DIABETIC CONTROL}

K. Khan, K. Ali, S. Farrukh, M. Haroon. Rheumatology, University Hospital Kerry, Tralee, County Kerry, Ireland

Background: Diabetes mellitus is one of the most common medical conditions all over the world. A variety of musculoskeletal (MSK) conditions have been associated with diabetes mellitus (DM). These MSK symptoms are important to recognise because these can respond to treatment. Estimates of the prevalence of MSK problems in patients with DM vary depending upon the definitions used for the problems and the study population with diabetes, which can range from a primary care cohort to patients with severe diabetes in a specialised referral setting.
Objectives: The objectives of our study were: 1) to investigate the prevalence of musculoskeletal complications of diabetes in a consecutive cohort of patients attending a secondary care endocrinology clinic; and 2) to identify clinical associations of such musculoskeletal complications by examining likely contributing features including patient characteristics, life style factors and features of underlying diabetic disease.

Methods: The study participants were all consecutive patients attending endocrinology clinics of University Hospital Kerry for the management of their DM. Patients with chronic inflammatory arthritis or chronic autoimmune diseases were also excluded. This was a questionnaire-based study and was carried out in two steps. The first step involved having a short interview and assessment of the patient, and in the second step their clinical records were reviewed to populate clinical parameters. The clinical variables studied were gender, smoking habits, body mass index (BMI), units of alcohol intake per week, smoking habits, type of DM, duration of DM, medications used for DM, employment status and also their current $\mathrm{HbA} 1 \mathrm{c}$ blood results. End organ complications of DM were also included Moreover, personal history of cardiovascular risk factors and diseases were also recorded, Since there is a long list of rheumatologic manifestations which are potentially associated with DM and many of them are less sensitive and specific for DM, we choose to study the 4 common DM-associated rheumatologic presentations (Stiff hands, Carpel Tunnel Syndrome, Charcot joint, bilateral shoulde rotator cuff tendinopathy). Moreover, importantly, only patients with the musculos keletal symptoms lasting for $>3$ months were included in the analysis. These 4 DM-associated musculoskeletal diseases are labelled as DM-MSK diseases. Results: A total of 250 patients [mean age $66 \pm 16$ years; $58 \%$ male; mean dura tion of DM $13 \pm 10$ years; mean BMI of $27.5 \pm 6$ ] were evaluated. DM-MSK diseases were present in $37.6 \%(n=94)$ of the entire cohort.

On univariate analysis, patients with older age, type-2 DM, using hypoglycaemic agents, hypertension, ischaemic heart disease, peripheral vascular disease, cerebrovascular accident, congestive heart disease, and patients with renal involvement had significantly higher prevalence of DM-MSK symptoms. However, on multivariate analysis (table-3), poor diabetic control as reflected by higher $\mathrm{HbAIC}$ levels, presence of diabetic kidney disease and advancing age were associated with the presence of DM-MSK symptoms.

Conclusions: We conclude that MSK manifestations of DM are very common but unfortunately these remain poorly recognised and continue to cause significant disabilities. Similar to the microvascular and macrovascular complications, MSK manifestations are associated with poor diabetic control.

Disclosure of Interest: K. Khan: None declared, K. Ali: None declared, S. Farrukh: None declared, M. Haroon Grant/research support from: abbvie, Pfizer, Speakers bureau: abbvie, Pfizer, UCB

DOI: 10.1136/annrheumdis-2018-eular.7598

\section{AB1153 IDIOPATIC LOBULAR PANNICULITIS (DISEASE WEBER- CHRISTIAN): CURRENT ASPECTS}

O.N. Egorova ${ }^{1}$, B.S. Belov ${ }^{1}$, S.I. Glukhova ${ }^{1}$, S.G. Radenska-Lopovok ${ }^{2}$. ${ }^{1}$ V. A Nasonova Research Institute of Rheumatology, ${ }^{2} 1$ FSBEIHE I.M. Sechenov Moscow State Medical University, Moscow, Russian Federation

Background: Idiopatic lobular panniculitis (ILP) (syn. Weber-Christian disease) is the least studied condition from the group of systemic connective tissue dis eases, which is characterised by predominant involvement of subcutaneous fat (SCF) tissue.

Objectives: To evaluate the interrelation between clinical signs and lab parameters in ILP patients.

Methods: The study evaluated 67 patients ( 9 males and 58 females) aged 20-76 years with verified ILP diagnosis and mean disease duration of $78.91^{48 ; 540}$ months who were on the record at V. A. Nasonova Research Institute of Rheumatology during 2007-2017 yy. Alfa-1 antitrypsin, liver fractions, amylase, lipase, trypsin ferritine, creatine phosphokinase (CPK), leptin and TNF- $\alpha$ levels were measured, chest CT and histopathological study of skin and SCF lesions biopsy specimens were made in addition to conventional clinical examination.

Results: ILP was found to affect all age groups, with $57 \%$ of cases falling on ablebodied adults aged 45-60 yy. Based on clinical manifestations including location, distribution, spatial extent of lesions, and clinical course of the disease, the following 4 clinical forms of ILP were identified: nodular (30 patients), plaque-like ${ }^{10}$ infiltrative $^{15}$ and mesenteric. ${ }^{12}$ ILP population demonstrated significant ESR $(p=0,01)$ and CRP $(p<0,0001)$ elevation. ESR elevation correlated with palpatory nodula pain intensity, assessed by visual analogue scale (VAS $(p<0,05, r=0,29)$, with the amount of affected body surface area (BSA) measured using the hand area surface (HAS) to equal $1 \%$ BSA $(p<0,05, r=0,50)$, with elevation of body temperature $(p<0,05, r=0,68)$ and $\operatorname{CRP}(p<0,05, r=0,68)$. CRP elevation correlated with pain intensity measured by VAS $(p<0,05, r=0,46)$, affected BSA $(p<0,05, r=0,61)$ and presence of stage II nodules $(p<0,05, r=0,41)$. Histopathologic features of skin and SCF biopsy specimens were studied in 65 patients $(97,01 \%)$, including antero - and retroperitoneal fat tissue biopsy specimens from 3 patients out of $5(5,97 \%)$ 